\title{
ON EXPECTED NUMBER OF LEVEL CROSSINGS OF A RANDOM HYPERBOLIC POLYNOMIAL
}

\author{
MINA KETAN MAHANTI AND LOKNATH SAHOO
}

\begin{abstract}
Let $g_{1}(\omega), g_{2}(\omega), \ldots, g_{n}(\omega)$ be independent and normally distributed random variables with mean zero and variance one. We show that, for large values of $n$, the expected number of times the random hyperbolic polynomial $y=g_{1}(\omega) \cosh x+g_{2}(\omega) \cosh 2 x+\cdots+g_{n}(\omega) \cosh n x$ crosses the line $y=L$, where $L$ is a real number, is $\frac{1}{\pi} \log n+O(1)$ if $L=o(\sqrt{n})$ or $L / \sqrt{n}=O(1)$, but decreases steadily as $O(L)$ increases in magnitude and ultimately becomes negligible when $n^{-1} \log L / \sqrt{n} \rightarrow \infty$.
\end{abstract}

1. Introduction. Let $g_{1}(\omega), g_{2}(\omega), \ldots, g_{n}(\omega)$ be normally distributed and independent random variables defined on a fixed probability space $(\Omega, A, P r)$ with mean zero and variance one. Consider the family of curves given by the random hyperbolic polynomial

$$
y=f_{n}(x)=\sum_{j=1}^{n} g_{j}(\omega) \cosh j x .
$$

The behavior of these curves to an extent can be understood by knowing their oscillations about different curves in the $\mathrm{x}-\mathrm{y}$ plane. Das [3] found out the expected number of oscillations of $f_{n}(x)$ about the x-axis and Farahmand [4] calculated its expected number of $L$-level crossings, i.e., oscillations about the line $y=L$ where $L$ does not exceed $O(\sqrt{n})$. Both of the estimates are asymptotic to $\frac{1}{\pi} \log n$ with an error term $\sqrt{\log n}$. It will be interesting to see how the L-level crossings change if $L$ exceeds $O(\sqrt{n})$. We show that the average number of L-level crossings of $f_{n}(x)$ decreases as $L$ increases beyond $O(\sqrt{n})$ and attains a specific value. The average number of oscillations about $y=L$ does not change if $L$ is

2010 AMS Mathematics subject classification. Primary 60H99, Secondary 26C99.

Keywords and phrases. Random polynomial, expected number of zeros, KacRice formula.

Received by the editors on July 13, 2013. 
larger than this specific value. In the sequel, we have also shown that the error term $\sqrt{\log n}$ should in fact be $O(1)$.

Let $E N_{n}(a, b)$ stand for the expected number of real zeros of $f_{n}(x)=$ $L$ in $(a, b)$. We prove the following theorem:

Theorem 1.1. If the coefficients of $f_{n}(x)$ in (1.1) are independent and normally distributed random variables with mean zero and variance one, then, for all sufficiently large values of $n$,

$$
E N_{n}(-\infty, \infty)=\left\{\begin{array}{c}
\frac{1}{\pi} \log n+O(1), \text { if } L=o(\sqrt{n}) \text { or } L=O(\sqrt{n}) ; \\
\frac{1}{\pi} \log \left(\frac{n}{\log h_{n}}\right)+O(1), \text { if } h_{n} \rightarrow \infty \text { but } \log h_{n}=o(n) ; \\
\frac{1}{\pi} \log \operatorname{coth}\left((4 n)^{-1} \log h_{n}\right)+1+O\left(n^{-1} \log n\right), \\
\quad \text { if } \log h_{n}=O(n) ; \\
1+o(1), \text { if } n^{-1} \log h_{n} \rightarrow \infty,
\end{array}\right.
$$

where $h_{n}=L / \sqrt{n}$.

The paper has been organized in the following manner. In Section 2, we discuss some preliminary concepts required for the proof of the theorem. We discuss the effect of different values of $L$, which are mentioned in the statement of the theorem, on the expected number of real zeros of $f_{n}(x)-L$ in Lemmas 3.1, 3.2 and 3.3 proved in Section 3. We first discuss some preliminary analysis required for the proof.

2. Preliminary analysis. Let us consider $f_{n}(x)$ as a non-stationary random process. The Kac-Rice formula [2] for the expected number of L-level crossings in $(a, b)$ of such a process is given by

$$
\begin{aligned}
E N_{n}(a, b)= & \int_{a}^{b} \frac{\sqrt{D_{n}}}{A_{n} \pi} e^{-\left(L^{2} C_{n}\right) /\left(2 D_{n}\right)} d x \\
& +\int_{a}^{b} \frac{L B_{n}}{\sqrt{2 \pi} A_{n}^{3 / 2}} \mathrm{e}^{-\left(L^{2}\right) /\left(2 A_{n}\right)} \phi\left(\frac{L B_{n}}{\sqrt{2 A_{n} D_{n}}}\right) d x \\
= & I_{1}(a, b)+I_{2}(a, b),
\end{aligned}
$$

where $A_{n}=\sum_{j=1}^{n} \cosh ^{2} j x, B_{n}=\sum_{j=1}^{n} j \cosh j x \sinh j x, C_{n}=$ $\sum_{j=1}^{n} j^{2} \sinh ^{2} j x, D_{n}=A_{n} C_{n}-B_{n}^{2}$ and $\phi(x)=(2 / \sqrt{\pi}) \int_{0}^{x} e^{-t^{2}} d t$ is the error function. Since the integrands in (2.1) are even functions of 
$x$, we note that

$$
E N_{n}(-\infty, \infty)=2 I_{1}(0, \infty)+2 I_{2}(0, \infty)
$$

Our aim will be to break up $(0, \infty)$ into suitable subintervals $(a, b)$ such that it will be possible to calculate $I_{1}(a, b)$ and $I_{2}(a, b)$ after obtaining the dominant terms of the respective integrands. Moreover, calculation of $I_{1}(0,1)$ will need special attention as it will provide the asymptotic estimate of $E N_{n}(0, \infty)$. The intervals where we can express $A_{n}, B_{n}$ and $C_{n}$ in terms of well-defined dominant terms are $\left(0,6 m^{-1} \log m\right)$ and $\left(06 m^{-1} \log m, \infty\right)$, where $m=2 n+1$. Using the dominant terms, we shall then obtain some useful results by means of which we can calculate the expected number of real zeros of $f_{n}(x)-L$ in the above mentioned two intervals in Section 3.

Consider the interval $\left(0,6 m^{-1} \log m\right)$. Let us write $A_{n}, B_{n}$ and $C_{n}$ in the following manner:

$$
4 A_{n}=m u(1+U), 8 B_{n}=m^{2} v(1+V), 48 C_{n}=m^{3} w(1+W),
$$

where $\lambda=m x, u=u(\lambda)=1+\lambda^{-1} \sinh \lambda, U=(-2+\tau \sinh \lambda)(m u)^{-1}$, $v=v(\lambda)=d u / d \lambda, V=v^{-1}\left(x \cosh \lambda+m^{-2} \tau^{\prime} \sinh \lambda\right), w=w(\lambda)=$ $3 d^{2} u / d \lambda^{2}-1, W=w^{-1}\left\{3 m^{-3} \tau^{\prime \prime} \sinh \lambda+m^{-2}\left(6 \tau^{\prime} \cosh \lambda+\cosh \lambda+\right.\right.$ $3 x \sinh \lambda)\}$ and $\tau=1 / \sinh x-1 / x$.

The validity of the above expressions can be verified from the definitions of $A_{n}, B_{n}$ and $C_{n}$ mentioned earlier. Note that $\tau$ can be represented as the following power series:

$$
\tau=\sum_{k=1}^{\infty}\left(2\left(1-2^{2 k-1}\right) \mathfrak{B}_{2 k} /(2 k) !\right) x^{2 k-1},
$$

where $\mathfrak{B}_{2 k}$ is the Bernoulli's number of degree $2 k$ [5]. The series converges absolutely and uniformly in $(0,1)$. Consequently $\tau, \tau^{\prime \prime}$ and $\tau^{\prime \prime \prime}$ are bounded in $(0,1)$, and it follows from definitions of $U, V$ and $W$ that in $\left(0,6 m^{-1}\right)$,

$$
U=O\left(m^{-1}\right), \quad V=O\left(m^{-2}\right), \quad W=O\left(m^{-3}\right) .
$$

On the other hand, if $x$ is in $\left(6 m^{-1}, 3 m^{-1} \log m\right)$, we observe that $U=$ $O\left(x e^{-\lambda}\right), V=O\left(x^{2}\right)$ and $W=O\left(x^{2}\right)$. Therefore, in $\left(0,3 m^{-1} \log m\right)$, we have

$$
A_{n}^{-1} \sqrt{12 D_{n}}=m \alpha_{n}(1+O(x / \sinh \lambda))
$$


and

$$
C_{n} / D_{n}=4(2 n+1)^{-1} \delta_{n}(1+O(x / \sinh \lambda))
$$

where

$$
\alpha_{n}=\alpha_{n}(\lambda)=u^{-1}\left(u w-3 v^{2}\right)^{1 / 2}, \quad \delta_{n}=\delta_{n}(\lambda)=w\left(u w-3 v^{2}\right)^{-1} .
$$

Thus, if $(a, b) \subset\left(0,3 m^{-1} \log m\right)$,

$$
I_{1}(a, b)=(2 \sqrt{3} \pi)^{-1} \int_{m a}^{m b} \alpha_{n} e^{-2 h_{n} \delta_{n}}\{1+O(x / \sinh \lambda)\} d \lambda .
$$

Since the integral in (2.3) is not amenable to direct integration, we need a suitable approximation of it. To this end, we need to calculate upper and lower bounds of $\alpha_{n}$ and $\delta_{n}$ in $\left(6 m^{-1}, 3 m^{-1} \log m\right)$. In this respect, the following inequality involving $\alpha_{n}$, obtainable after applying a little algebra, will be useful:

$$
\frac{\lambda^{2}-3 \lambda-3}{3 \sinh \lambda}<\frac{\alpha_{n}}{\sqrt{3}}-\frac{1}{\lambda}<\frac{\lambda^{2}-3 \lambda+3}{3 \sinh \lambda} .
$$

We also note that $\delta_{n}$ can be written in the following manner:

$$
\delta_{n}=\left(\lambda^{3} / \sinh \lambda\right)\left(p_{1} / p_{2}\right),
$$

where $p_{1}=w \lambda / \sinh \lambda$ and $p_{2}=\lambda^{4}\left(u w-3 v^{2}\right) \sinh ^{-2} \lambda$. From the derivatives of $p_{1}$ and $p_{2}$ we notice that $p_{1}$ is a monotonically increasing function and $p_{2}$ is a monotonically decreasing function of $x$ in $\left(6 m^{-1}, \infty\right)$. Using this information, we find that, in $\left(6 m^{-1}, 3 m^{-1} \log m\right)$,

$$
0.5 \lambda^{3} / \sinh \lambda<\delta_{n}<\left(\lambda^{3} / \sinh \lambda\right) .
$$

As a consequence, in $(a, b)$, we have

$$
\mathrm{e}^{-\left(2 h_{n}(a m)^{3}\right) /(\sinh (a m))} \leq \mathrm{e}^{-2 h_{n} \delta_{n}(\lambda)} \leq \mathrm{e}^{-\left(h_{n}(b m)^{3}\right) /(\sinh (b m))} .
$$

With the help of this inequality and (2.4), we conclude that

$$
\begin{aligned}
& e^{-2 h_{n}(a m)^{3} / \sinh (a m)}\left[\log \frac{b}{a}+\int_{m a}^{m b} \frac{\lambda^{2}-3 \lambda-3}{3 \sinh \lambda} d \lambda\right]\left(1+O\left(n^{-1}\right)\right) \\
& \leq 2 \pi I_{1}(a, b) \\
& \leq e^{-2 h_{n}(b m)^{3} / \sinh (b m)}\left[\log \frac{b}{a}+\int_{m a}^{m b} \frac{\lambda^{2}-3 \lambda+3}{3 \sinh \lambda} d \lambda\right]\left(1+O\left(n^{-1}\right)\right) .
\end{aligned}
$$


It is to be noted that $I_{1}\left(6 m^{-1}, 3 m^{-1} \log m\right)$ can be calculated with the help of (2.6) if $h_{n} \rightarrow \infty$. But if $h_{n} \rightarrow 0$ or $h_{n}=O(1)$, we shall have to use $(2.7)$, which can be obtained by integration by parts and using the bounds of $\alpha_{n}$ and $\delta_{n}$ that have been mentioned above

$$
\begin{aligned}
& \frac{1}{\sqrt{3}} \int \alpha_{n} e^{-2 h_{n} \delta_{n}} d \lambda=\log \lambda e^{-2 h_{n} \delta_{n}} \\
& \quad+2 \xi_{1}\left[h_{n} \delta_{n} \log \lambda+\int\left\{\left(\frac{\alpha_{n}}{\sqrt{3}}-\frac{1}{\lambda}\right)-\frac{h_{n} \xi_{2} \lambda^{2}}{\sinh \lambda} d \lambda\right\}\right]
\end{aligned}
$$

where $e^{-h_{n} \delta(6)} \leq \xi_{1}<1$ and $0.5 \leq \xi_{2}<1$.

Let us now consider the interval $\left(3 m^{-1} \log m, \infty\right)$. In this interval, for all sufficiently large $n, n^{s} \sinh ^{s} x(\sinh n x)^{-1}$, where $s$ is a finite positive number, a monotonically decreasing function of $x$ and tends to zero for sufficiently large values of $n$. Therefore, the following relations are valid at $x=a$, where $a \geq 3 m^{-1} \log m$ :

$$
\begin{aligned}
4 A_{n} & =g_{m}(x)\left(1+O\left(a m e^{-a m}\right)\right), \\
8 B_{n} & =g_{m}(x)\left(m-\operatorname{coth} x+O\left(n e^{-2 a m}\right)\right), \\
16 C_{n} & =g_{m}(x)\left(m^{2}+1-2 m \operatorname{coth} x+2 \cos e c h^{2} x+O\left(n^{3} a e^{-a m}\right)\right) .
\end{aligned}
$$

The $O($ ) terms decrease in magnitude as $x$ increases. Hence, in any interval $(a, b) \subset\left(3 m^{-1} \log m, \infty\right)$, we have

$$
I_{1}(a, b)=(2 \pi)^{-1} \xi_{3} \int_{a}^{b}(\sinh x)^{-1}\left(1+O\left(n^{2} p_{n} e^{-p_{n}}\right)\right) d x,
$$

where

$$
e^{-2 h_{n}(m \sinh a)^{3} / \sinh m a}<\xi_{3}<e^{-2 h_{n}(m \sinh b)^{3} / \sinh m b} .
$$

We are interested in determining how $E N_{n}(-\infty, \infty)$ changes with a change in magnitude of $L$. The relationship between $E N_{n}(-\infty, \infty)$ and $L$ can be fully established by considering four different ranges of values of $L$. In Lemma 3.1 we show that the asymptotic value of $L$ level crossings of $f_{n}(x)$ remains fixed at $\pi^{-1} \log n$ if $L=o\left(n^{1 / 2}\right)$ or $L=O\left(n^{1 / 2}\right)$. In Lemma 3.2 and Lemma 3.3 we show that the number of crossings starts decreasing as $L$ increases in value, but becomes stationary beyond a value of $L$ mentioned in Lemma 3.3. 


\section{Proof of the theorem.}

\section{Lemma 3.1.}

$$
\begin{aligned}
& E N_{n}(-\infty, \infty) \\
= & \left\{\begin{array}{l}
\pi^{-1}\left[\log m+l_{1}+l_{2}+2 \pi l_{4}+\log 2\right]\left(1+O\left(n^{-1}\right)\right) \text { if } h_{n}=O(1), \\
\pi^{-1}\left[\log m+1.28665+2 l_{3}+\log 2\right]\left(1+O\left(n^{-1}\right)\right) \text { if } h_{n}=o(1),
\end{array}\right.
\end{aligned}
$$

where $l_{1}, l_{2}, l_{3}$ and $l_{4}$ are constants independent of $n$ mentioned in (3.1), (3.3) and (3.5).

Proof. Let us assume that $h_{n}=O(1)$. The intervals that need to be considered to calculate $I_{1}(0, \infty)$ in this case are $\left(0,6 m^{-1}\right)$, $\left(6 m^{-1}, 3 m^{-1} \log m\right)$ and $\left(3 m^{-1} \log m, \infty\right)$.

Consider the interval $\left(0,6 \mathrm{~m}^{-1}\right)$ first. It can be seen from the definitions of $u, v$ and $w$ that they are finite in $\left(0,6 m^{-1}\right)$ and, since $u$ does not vanish, $\alpha_{n}$ is bounded. If we take the derivative of $w\left(u w-3 v^{2}\right)^{-1}$ with respect to $\lambda$, we find that $\delta_{n}$ is a monotonically decreasing function of $\lambda$. Therefore, by (2.3), we have

$$
I_{1}(0,6 / m)=l_{1},
$$

where $l_{1}$ is a constant and is given by

$$
\exp \left(-9 h_{n} / 2\right) \int_{0}^{6} \alpha_{n} d \lambda<2 \pi \sqrt{3} l_{1}<\exp \left(-2 h_{n} \delta_{n}(6)\right) \int_{0}^{6} \alpha_{n} d \lambda
$$

Consider the interval $\left(6 m^{-1}, 3 m^{-1} \log m\right)$ now. It follows from $(2.3)$ and (2.7) that

$$
I_{1}\left(6 m^{-1}, 3 m^{-1} \log m\right)=(2 \pi)^{-1}\left[\log \log m+l_{2}\right](1+O(1 / n)),
$$

where $l_{2}$ is a constant and can be calculated using numeric integration and (2.5) as

$l_{2}=\log 3-(\log 6) e^{-2 h_{n} \delta_{n}(6)}-2 \xi_{1}\left[h_{n}\left\{(\log 6) \delta_{n}(6)+0.247876 \xi_{2}\right\}-l_{3}\right]$, where $0.0528801<l_{3}<.0429651$.

We can find $I_{1}\left(3 m^{-1} \log m, \infty\right)$ using $(2.8)$ as

$$
I_{1}\left(3 m^{-1} \log m, \infty\right)=(2 \pi)^{-1}(\log (m / \log m)-\log 1.5)+O\left(n^{-1} \log n\right)^{2} .
$$


We now turn our attention to the calculation of $I_{2}(0, \infty)$. Let $L / \sqrt{2 A_{n}}$ in the integrand of $I_{2}(a, b)$ be substituted by $s$. It follows immediately that

$$
I_{2}\left(3 m^{-1} \log m, \infty\right)<\int_{0}^{O\left(m^{-7} \log m\right)} e^{-s^{2}} d s=O\left(m^{-7} \log m\right) .
$$

In order to calculate $I_{2}\left(0,3 m^{-1} \log m\right)$, we need to estimate a lower bound and an upper bound of it. To obtain the lower bound, we first notice from the definition of $u, v$ and $w$ that $3 v^{2}<u w$ in $\left(0,6 m^{-1} \log m\right)$. Since $\delta_{n}$ is a monotonically decreasing function of $\lambda$, we also find that $v_{2}^{2} /\left\{u_{1}\left(u_{1} w_{3}-3 v_{2}^{2}\right)\right\}<\delta_{n} / 3<3 / 8$. As a consequence,

$$
\frac{L^{2} B_{n}^{2}}{2 A_{n} D_{n}}=\frac{6 h_{n} v_{2}^{2}}{u_{1}\left(u_{1} w_{3}-3 v_{2}^{2}\right)}\left(1+O\left(\frac{x}{\sinh \lambda}\right)\right)<\frac{9 h_{n}}{4}\left(1+O\left(\frac{x}{\sinh \lambda}\right)\right) .
$$

Using the above inequality, we conclude that

$$
I_{2}\left(0,3 m^{-1} \log m\right)<\pi^{-1 / 2} \phi\left(3 \sqrt{h_{n}} / 2\right) \int_{0}^{h_{n} / \sqrt{2}} \exp \left(-s^{2}\right) d s .
$$

It is also easy to verify that $u_{1} w_{3}<6 v_{2}^{2}$. Therefore, for large values of $n, B_{n}^{2} / D_{n}>1$, and consequently,

$$
\pi^{-1 / 2} \int_{0}^{h_{n} / \sqrt{2}} \exp \left(-s^{2}\right) \phi(s) d s+O\left(n^{-1}\right)<I_{2}\left(0,3 m^{-1} \log m\right) .
$$

It follows from the last two inequalities that

$$
I_{2}\left(0,3 m^{-1} \log m\right)=l_{4}+O\left(n^{-1}\right),
$$

where $l_{4}$ is a constant given by

$$
\phi\left(h_{n}^{2} / 2\right) / 4<l_{4}<\phi\left(3 \sqrt{h_{n}} / 2\right) \phi\left(h_{n} / \sqrt{2}\right) / 2 .
$$

The proof of Lemma 3.1 for $h_{n}=O(1)$ is thus obtained from (3.1)-(3.5) and (2.2).

In order to calculate $E N_{n}(-\infty, \infty)$ for the case $h_{n}=o(1)$, we need only to let $h_{n} \rightarrow 0$ in (2.2) and (3.1)-(3.5). We can find the approximate value of $\int_{0}^{6} \alpha_{n} d \lambda$ to be 2.22854 by applying Simpson's $1 / 3$ rule. Thus, we find that the claim in Lemma 3.1 is true for the case $h_{n}=o(1)$. 
Lemma 3.2. If $h_{n} \rightarrow \infty$ as $n \rightarrow \infty$, but $\log h_{n}=o(n)$, then for large values of $n$,

$$
E N_{n}(-\infty, \infty)=\pi^{-1}\left\{\log \left(m / \log h_{n}\right)+\log 2\right\}+1+o(1) .
$$

Proof. We first calculate $I_{1}(0, \infty)$. Let us recall that $\alpha_{n}$ is bounded and $\delta_{n}$ is a decreasing function in $\left(0,6 m^{-1}\right)$. Therefore, by $(2.3)$ and for all values of $h_{n}$ satisfying the condition of Lemma 3.2, we have

$$
I_{1}(0,6 / m)=O\left(\exp \left(-2 h_{n} \delta_{n}(6)\right)\right) .
$$

To calculate $\left(6 m^{-1}, \infty\right)$, it is necessary to distinguish between two cases, i.e., whether $h_{n}>O\left(\mathrm{~m}^{3}\right)$ or $h_{n} \leq O\left(\mathrm{~m}^{3}\right)$. In the former case, we find by (2.6) that

$$
I_{1}\left(6 m^{-1}, 3 m^{-1} \log m\right)=O\left(\log \log m e^{-2 h_{n}\left(m^{-1} \log m\right)^{3}}\right),
$$

and by $(2.8)$, we find that

$$
\begin{aligned}
& I_{1}\left(3 m^{-1} \log m, m^{-1} \log h_{n}\right)=O\left(\log \log h_{n} e^{-\left(\log h_{n}\right)^{3}}\right), \\
& I_{1}\left(m^{-1} \log h_{n}, m^{-1}\left(\log h_{n}+5 \log \log h_{n}\right)\right)=O\left(\log \log h_{n} / \log h_{n}\right), \\
& \quad I_{1}\left(m^{-1}\left(\log h_{n}+5 \log \log h_{n}\right), \infty\right)=(2 \pi)^{-1}\left\{\log \left(m / \log h_{n}\right)+\log 2\right\} \\
& \quad+O\left\{\log n /\left(\log h_{n}\right)^{2}\right\} .
\end{aligned}
$$

Therefore, if $h_{n}>O\left(m^{3}\right)$, we conclude that

$$
I_{1}(0, \infty)=(2 \pi)^{-1}\left\{\log \left(m / \log h_{n}\right)+\log 2\right\}+O\left(\log n /\left(\log h_{n}\right)^{2}\right) .
$$

Let us now consider the case when $h_{n} \leq O\left(\mathrm{~m}^{3}\right)$. We observe from (2.6) that, in this case,

$$
I_{1}\left(6 m^{-1}, m^{-1} \log h_{n}\right)=O\left(\log \log h_{n} e^{-\left(\log h_{n}\right)^{3}}\right) .
$$

To calculate $I_{1}(a, b)$ in other subintervals, we have to consider two cases again, i.e., $h_{n}(\log m)^{3}(\log \log m)^{2} \leq O\left(m^{3}\right)$ and $h_{n}(\log m)^{3}(\log \log m)^{2}$ $>O\left(\mathrm{~m}^{3}\right)$. Let us consider the former case first. We then obtain from (2.6) that

$$
\begin{aligned}
I_{1}\left(m^{-1} \log h_{n}, m^{-1}\left(\log h_{n}+3 \log \log h_{n}+\right.\right. & \left.\left.2 \log \log \log h_{n}\right)\right) \\
& =O\left(\log \log h_{n} / \log h_{n}\right),
\end{aligned}
$$




$$
\begin{aligned}
& I_{1}\left(m ^ { - 1 } \left(\log h_{n}+\right.\right.\left.\left.3 \log \log h_{n}+2 \log \log \log h_{n}\right), 3 m^{-1} \log m\right) \\
&=(2 \pi)^{-1} \log \left(3 \log m / \log h_{n}\right)+O\left(1 /\left(\log \log h_{n}\right)\right) .
\end{aligned}
$$

By (2.8), we have

$$
\begin{aligned}
& I_{1}\left(3 m^{-1} \log m, \infty\right) \\
& \quad=(2 \pi)^{-1}\{\log (m / \log m)-\log 1.5\}+O(\log \log m / \log m) .
\end{aligned}
$$

Therefore, if $h_{n} \leq O\left(m^{3}\right)$ and $h_{n}(\log m)^{3}(\log \log m)^{2} \leq O\left(m^{3}\right)$, we obtain that

$$
I_{1}(0, \infty)=(2 \pi)^{-1}\left\{\log \left(m / \log h_{n}\right)+\log 2\right\}+O(\log \log m / \log m)
$$

Let us now consider the case when $h_{n}<O\left(m^{3}\right)<h_{n}(\log m)^{3}(\log \log m)^{2}$. It can be seen from (2.6) that, in this case,

$$
\begin{aligned}
& I_{1}\left(m^{-1} \log h_{n}, 3 m^{-1}(\log m-\log \log \log m)\right)=O\left((\log m)^{-1}\right) \\
& I_{1}\left(3 m^{-1}(\log m-\log \log \log m), 3 m^{-1} \log m\right)=O(\log \log m / \log m) .
\end{aligned}
$$

Also, by (2.8), we obtain that

$$
\begin{aligned}
& I_{1}\left(3 m^{-1} \log m, m^{-1}\left(\log h_{n}+3 \log \log m+2 \log \log \log m\right)\right) \\
& <I_{1}\left(3 m^{-1} \log m, m^{-1}(\log m+3 \log \log m+2 \log \log \log m)\right) \\
& =O(\log \log m / \log m), \\
& I_{1}\left(m^{-1}\left(\log h_{n}+3 \log \log m+2 \log \log \log m\right), \infty\right) \\
& =(2 \pi)^{-1}\left\{\log \left(m / \log h_{n}\right)+\log 2\right\} \\
& \quad+o(\log \log m / \log m) .
\end{aligned}
$$

Therefore, if $h_{n} \leq O\left(m^{3}\right)$ and $h_{n}(\log m)^{3}(\log \log m)^{2}>O\left(m^{3}\right)$, we observe that (3.8) is also satisfied.

We now calculate $I_{2}(0, \infty)$. By taking $s=L / \sqrt{2 A_{n}}$, we find that

$$
\begin{gathered}
I_{2}\left(0, \log h_{n} / n\right)<\pi^{-1 / 2}\left\{\phi\left(h_{n} / \sqrt{2}\right)-\phi\left(\sqrt{2 \log h_{n}}\right)\right\}=O\left(\frac{e^{-2 \log h_{n}}}{\sqrt{\log h_{n}}}\right), \\
I_{2}\left(\frac{3 \log h_{n}}{2 n}, \infty\right)<\pi^{-1 / 2} \phi\left(\sqrt{\frac{3 \log h_{n}}{h_{n}}}\right)=O\left(\sqrt{h_{n}^{-1} \log h_{n}}\right) .
\end{gathered}
$$


Let $\varphi(a, b, c)$ represent the integral $\int_{a}^{b} \pi^{-1 / 2} e^{-s^{2}} \phi(c s) d s$. Note that $B_{n} / \sqrt{D_{n}}=\lambda\left(1+O\left(1 / \log h_{n}\right)\right)$ if $x>m^{-1} \log h_{n}$. So

$$
\begin{aligned}
\varphi\left(\sqrt{\frac{3 \log h_{n}}{h_{n}}}, \sqrt{2 \log h_{n}}, 2 \log h_{n}\right) & <I_{2}\left(\log h_{n} / n, 3 \log h_{n} /(2 n)\right) \\
& <\varphi\left(\sqrt{\frac{3 \log h_{n}}{h_{n}}}, \sqrt{2 \log h_{n}}, 3 \log h_{n}\right) .
\end{aligned}
$$

Since $\varphi(0, \infty, c)=\pi^{-1 / 2} \arctan c$, by the last inequality, we obtain

$$
I_{2}\left(\log h_{n} / n, 3 \log h_{n} /(2 n)\right)=1 / 2+O\left(h_{n}^{-1} \log h_{n}\right)^{1 / 2} .
$$

It now follows that

$$
I_{2}(0, \infty)=1 / 2+o(1)
$$

Validity of Lemma 3.2 now follows from (3.7)-(3.9).

Lastly, we settle the case $\log h_{n} \geq O(n)$ in the following lemma.

Lemma 3.3. Let $m^{-1} \log h_{n}=q_{n}+o(1)$, Then,

$$
\begin{aligned}
& E N_{n}(-\infty, \infty) \\
& =\left\{\begin{array}{l}
\pi^{-1} \log \operatorname{coth}\left(q_{n} / 2\right)+1+O\left(n^{-1} \log n\right) \\
\text { if } q_{n} \text { is a constant }, \\
1+o(1) \quad \text { if } q_{n} \rightarrow \infty \text { as } n \rightarrow \infty
\end{array}\right.
\end{aligned}
$$

Proof. It is easy to see that (3.6) is true. Using (2.6) and (2.8), we find that

$$
\begin{aligned}
I_{1}\left(6 m^{-1}, 3 m^{-1} \log m\right) & =O\left(\log \log n \exp \left(-h_{n}\left(n^{-1} \log n\right)^{3}\right)\right), \\
I_{1}\left(3 m^{-1} \log m, q_{n}\right) & =O\left(e^{-2\left(m \sinh q_{n}\right)^{3}} \log n\right) .
\end{aligned}
$$

By (2.8), we obtain the following estimates if $q_{n}$ is a finite constant:

$$
\begin{aligned}
I_{1}\left(q_{n}, q_{n}+\left(4 \log n+3 q_{n}\right) / m\right)= & O\left(n^{-1} \log n\right), \\
I_{1}\left(q_{n}+\left(4 \log n+3 q_{n}\right) / m, \infty\right)= & (2 \pi)^{-1} \log \operatorname{coth}\left(q_{n} / 2\right) \\
& +O\left(n^{-1} \log n\right) .
\end{aligned}
$$


On the other hand, if $q_{n} \rightarrow \infty$ as $n \rightarrow \infty$, we find that

$$
I_{1}\left(q_{n}, \infty\right)=O\left(e^{-2\left(m \sinh q_{n}\right)^{3}}\right) .
$$

From the above discussion and (3.6), we find that

$$
\begin{aligned}
& \text { (3.10) } I_{1}(0, \infty) \\
& =\left\{\begin{array}{l}
(2 \pi)^{-1} \log \operatorname{coth}\left(q_{n} / 2\right)+O\left(n^{-1} \log n\right) \text { if } q_{n} \text { is a finite constant; } \\
O\left(e^{-2 q_{n}}\right) \text { if } q_{n} \rightarrow \infty \text { as } n \rightarrow \infty
\end{array}\right.
\end{aligned}
$$

$I_{2}(0, \infty)$ can be calculated in a manner similar to that in Lemma 3.2. We observe that $Y_{n} / \sqrt{D_{n}}=m \sinh x(1+o(1))$ if $x>\log h_{n} / 2 n$. Then

$$
\begin{aligned}
& I_{2}\left(0, \log h_{n} / n\right)<O\left(e^{-2 n h_{n}^{1 / n}} /\left(\sqrt{n} h_{n}^{1 / n}\right)\right), \\
& I_{2}\left(3 \log h_{n} /(2 n), \infty\right)<O\left(\sqrt{n} h_{n}^{(3-n) /(2 n)}\right) \text {. } \\
& \varphi\left(\sqrt{2 n h_{n}^{(3-2 n) /(2 n)}}, \sqrt{2 n h_{n}^{1 / n}}, n h_{n}^{1 / n}\right)<I_{2}\left(\log h_{n} / n, 3 \log h_{n} /(2 n)\right) \\
& <\varphi\left(\sqrt{2 n h_{n}^{(3-2 n) /(2 n)}}, \sqrt{2 n h_{n}^{1 / n}}, n h_{n}^{3 /(2 n)}\right) .
\end{aligned}
$$

It follows that

$$
I_{2}(0, \infty)=1 / 2+o(1) .
$$

We obtain the proof of Lemma 3.3 from (3.10) and (3.11).

The proof of the theorem follows from Lemmas 3.1, 3.2 and 3.3.

\section{REFERENCES}

1. A.T. Bharucha-Reid and M. Sambandham, Random polynomials, Academic Press, Orlando, 1986.

2. H. Crammer and A.R. Leadbetter, Stationary and related stochastic process, Wiley, New York, 1967.

3. M. Das, On real zeros of random polynomial with hyperbolic elements, unpublished Ph.D dissertation, Utkal University, India, 1971.

4. K. Farahamand, Level crossings of random polynomial with hyperbolic elements, Proc. Amer. Math. Soc. 123 (1887)-(1892), 1995.

5. I.S. Gradshtyn and I.M. Ryzhik, Tables of integrals, Series and Products, Academic Press, New York, 1980. 
College of Basic Science and Humanities, Department of Mathematics, OUAT, BHUBANESWAR, Odisha, 751003, India

Email address: minaketan_mahanti@yahoo.com

Gopabandhu Science College, Atagarh, Odisha, 751003, India

Email address: loknath.math@gmail.com 